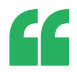

[islet-kidney] transplantation reversed diabetic hyperglycaemia and restored renal function

TRANSPLANTATION

\title{
A new tolerance strategy for diabetic recipients
}

A novel approach involving transplantation of composite islet-kidney (IK) grafts in combination with tolerance induction could potentially become an attractive treatment option for diabetic patients with end-stage renal disease (ESRD) say researchers. Kazuhiko Yamada and colleagues report that this strategy enabled graft acceptance and reduced the need for insulin therapy in a Rhesus macaque model.

"Many diabetic patients with renal failure have potential donors who are willing to provide a kidney and islets, but the quantity of islets necessary to achieve insulin independence hampers successful islet transplantation by partial pancreatectomy from living donors," explains Yamada. "In addition, transplant recipients have to endure the risks associated with immunosuppression for the rest of their lives. In order for successful islet transplantation using living donors, innovative strategies must be developed to overcome these major issues."

Yamada and colleagues previously showed that IK transplantation reversed diabetic hyperglycaemia and restored renal function in baboons receiving chronic immunosuppression. To generate the IKs, islets were isolated from the pancreas and injected under the renal capsule of the donor at least 2 months before transplantation. Yamada comments that by enabling engraftment of the islets in an autologous kidney, this technique eliminates the need for islet engraftment in the allogeneic immune environment of the recipient so greatly improves islet survival.

In their new study, the researchers aimed to develop a clinically applicable tolerance-inducing regimen for diabetic patients with ESRD undergoing living-donor transplantation. To induce tolerance they transplanted four diabetic macaques with haematopoietic stem cells from related donors (using a novel reduced intensity protocol developed by C. Huang) 3 weeks before transplanting IKs from the same donors. After IK transplantation, three of the recipient macaques required only minimal doses of insulin, whereas the fourth

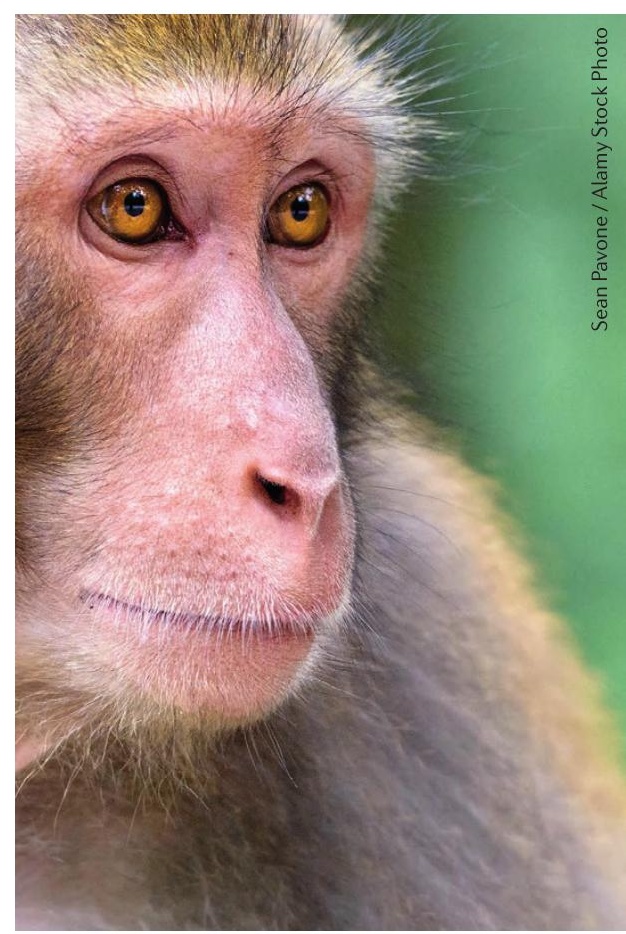

no longer required insulin therapy. All of the macaques accepted their grafts even after cessation of immunosuppression. "These data demonstrate proof of principle for our approach of inducing tolerance to islets and kidneys in living donor transplantation," concludes Yamada. Ellen F. Carney

ORIGINAL ARTICLE Pathiraja, V. et al. Tolerance of vascularized islet-kidney transplants in Rhesus monkeys. Am.J. Transplant. http://dx.doi. org/10.1111/ajt.13952 (2016) 